\title{
Determination of Concentration-Dependent Gas Diffusivity in Reservoir- Fluid Systems
}

\author{
Hyun Woong Jang and Daoyong Yang* \\ Petroleum Systems Engineering, Faculty of Engineering and Applied Science, \\ University of Regina, 3737 Wascana Parkway, Regina, SK S4S 0A2, Canada \\ *Corresponding author: Dr. Daoyong Yang \\ Tel: 1 (306) 337-2660 \\ Fax: 1 (306) 585-4855 \\ E-mail: Tony.Yang@uregina.ca
}




\section{Derivation of FEM Equation for $N$-element System}

To integrate the right-hand sides of Equations [22]-[24] using Equations [17a] and [17b], following matrices are obtained with corresponding elements in them.

$$
\begin{aligned}
& \boldsymbol{L}^{e, s}=\frac{A^{e, s}}{2 h}\left[\begin{array}{rr}
\left(x_{a}^{e}+x_{b}^{e}\right) & -\left(x_{a}^{e}+x_{b}^{e}\right) \\
-\left(x_{a}^{e}+x_{b}^{e}\right) & \left(x_{a}^{e}+x_{b}^{e}\right)
\end{array}\right] \\
& \boldsymbol{M}^{e, s}=\frac{B^{e, s}}{h}\left[\begin{array}{rr}
1 & -1 \\
-1 & 1
\end{array}\right] \\
& \boldsymbol{E}^{e}=\frac{h}{6}\left[\begin{array}{ll}
2 & 1 \\
1 & 2
\end{array}\right]
\end{aligned}
$$

Due to the nature of $\psi_{1}^{e}$ and $\psi_{2}^{e}$,

$$
\boldsymbol{Q}^{e, s}=\left[\begin{array}{l}
Q_{1}^{e, s} \\
Q_{2}^{e, s}
\end{array}\right]
$$

Because $A^{e, s}$ and $B^{e, s}$ are time-dependent values, matrices $\boldsymbol{L}^{e, s}$ and $\boldsymbol{M}^{e, s}$ are also time-dependent. Therefore, Reddy's ${ }^{37}$ original equation is modified to yield Equation [S.5] which is suitable for the current system.

$$
\left(\boldsymbol{L}^{e, s}+\boldsymbol{M}^{e, s}\right) \boldsymbol{c}^{e, s}+\boldsymbol{E}^{e} \dot{\boldsymbol{c}}^{e, s}=\boldsymbol{Q}^{e, s}
$$

where

$$
\begin{gathered}
\boldsymbol{c}=\left[\begin{array}{c}
c_{1}^{e, s} \\
c_{2}^{e, s}
\end{array}\right] \\
\dot{\boldsymbol{c}}=\left[\begin{array}{l}
\dot{c}_{1}^{e, s} \\
\dot{c}_{2}^{e, s}
\end{array}\right]
\end{gathered}
$$


For a $N$-element system in FEM, shown by Figure 1(b),

$$
\boldsymbol{L}^{S}=\frac{1}{2 h}\left[\begin{array}{cccc}
A^{e 1, s}\left(x_{1}^{e 1}+x_{2}^{e 1}\right) & -A^{e 1, s}\left(x_{1}^{e 1}+x_{2}^{e 1}\right) & 0 & 0 \\
-A^{e 1, s}\left(x_{1}^{e 1}+x_{2}^{e 1}\right) & A^{e 1, s}\left(x_{1}^{e 1}+x_{2}^{e 1}\right)+A^{e 2, s}\left(x_{1}^{e 2}+x_{2}^{e 2}\right) & -A^{e 2, s}\left(x_{1}^{e 2}+x_{2}^{e 2}\right) & 0 \\
0 & -A^{e 2, s}\left(x_{1}^{e 2}+x_{2}^{e 2}\right) & A^{e 2, s}\left(x_{1}^{e 2}+x_{2}^{e 2}\right)+A^{e 3, s}\left(x_{1}^{e 3}+x_{2}^{e 3}\right) & -A^{e 3, s}\left(x_{1}^{e 3}+x_{2}^{e 3}\right) \\
\ddots & \ddots & \ddots & \ddots \\
0 & 0 & 0 & -A^{e(N-2), s}\left(x_{1}^{e(N-2)}+x_{2}^{e(N-2)}\right) \\
0 & 0 & 0 & 0
\end{array}\right.
$$

$$
\left.\begin{array}{ccc}
0 & 0 & 0 \\
0 & 0 & 0 \\
0 & 0 & 0 \\
A^{e(N-2), s}\left(x_{1}^{e(N-2)}+x_{2}^{e(N-2)}\right)+A^{e(N-1), s}\left(x_{1}^{e(N-1)}+x_{2}^{e(N-1)}\right) & \ddots & 0 \\
-A^{e(N-1), s}\left(x_{1}^{e(N-1)}+x_{2}^{e(N-1)}\right) & A^{e(N-1), s}\left(x_{1}^{e(N-1)}+x_{2}^{e(N-1)}\right) & 0 \\
0 & -A^{e N, s}\left(x_{1}^{e N}+x_{2}^{e N}\right) & A^{e N, s}\left(x_{1}^{e N}+x_{2}^{e N}\right) \\
& & \left.A_{1}^{e N}+x_{2}^{e N}\right)
\end{array}\right]
$$

where $e n$ refers to the $n$th element, and $s$ refers to the $s$ th temporal step, $t_{s}$. 
As $x_{1}=x_{1}^{e 1}=0, x_{n}=x_{1}^{e n}=x_{2}^{e(n-1)}$ for $n \geq 2$, and $h=x_{2}^{e n}-x_{1}^{e n}$, Equation [S.7] reduces to Equation [S.8] as follows.

$\boldsymbol{L}^{s}=\frac{1}{2}\left[\begin{array}{cccc}A^{e 1, s} & -A^{e 1, s} & 0 & 0 \\ -A^{e 1, s} & A^{e 1, s}+3 A^{e 2, s} & -3 A^{e 2, s} & 0 \\ 0 & -3 A^{e 2, s} & 3 A^{e 2, s}+5 A^{e 3, s} & -5 A^{e 3, s} \\ \ddots & \ddots & \ddots & \ddots \\ 0 & 0 & 0 & -(2 N-5) A^{e(N-2), s} \\ 0 & 0 & 0 & 0 \\ 0 & 0 & 0 & 0\end{array}\right.$

$$
\left.\begin{array}{ccc}
0 & 0 & 0 \\
0 & 0 & 0 \\
0 & 0 & 0 \\
\ddots & \ddots & \ddots \\
(2 N-5) A^{e(N-2), s}+(2 N-3) A^{e(N-1), s} & -(2 N-3) A^{e(N-1), s} & 0 \\
-(2 N-3) A^{e(N-1), s} & (2 N-3) A^{e(N-1), s}+(2 N-1) A^{e N, s} & -(2 N-1) A^{e N, s} \\
0 & -(2 N-1) A^{e N, s} & (2 N-1) A^{e N, s}
\end{array}\right]
$$

Matrices $\boldsymbol{M}^{s}$ and $\boldsymbol{E}$ at temporal step $t_{s}$ for the $N$-element system are expressed as,

$\boldsymbol{M}^{s}=\frac{1}{h}\left[\begin{array}{ccccccc}B^{e 1, s} & -B^{e 1, s} & 0 & 0 & 0 & 0 & 0 \\ -B^{e 1, s} & B^{e 1, s}+B^{e 2, s} & -B^{e 2, s} & 0 & 0 & 0 & 0 \\ 0 & -B^{e 2, s} & B^{e 2, s}+B^{e 3, s} & -B^{e 3, s} & 0 & 0 & 0 \\ \ddots & \ddots & \ddots & \ddots & \ddots & \ddots & \ddots \\ 0 & 0 & 0 & -B^{e(N-2), s} & B^{e(N-2), s}+B^{e(N-1), s} & -B^{e(N-1), s} & 0 \\ 0 & 0 & 0 & 0 & -B^{e(N-1), s} & B^{e(N-1), s}+B^{e N, s} & -B^{e N, s} \\ 0 & 0 & 0 & 0 & 0 & -B^{e N, s} & B^{e N, s}\end{array}\right]$




$$
\boldsymbol{E}=\frac{h}{6}\left[\begin{array}{ccccccc}
2 & 1 & 0 & 0 & 0 & 0 & 0 \\
1 & 4 & 1 & 0 & 0 & 0 & 0 \\
0 & 1 & 4 & 1 & 0 & 0 & 0 \\
\ddots & \ddots & \ddots & \ddots & \ddots & \ddots & \ddots \\
0 & 0 & 0 & 1 & 4 & 1 & 0 \\
0 & 0 & 0 & 0 & 1 & 4 & 1 \\
0 & 0 & 0 & 0 & 0 & 1 & 2
\end{array}\right]
$$

The vectors $\boldsymbol{c}^{s}, \dot{\boldsymbol{c}}^{s}$ and $\boldsymbol{Q}^{s}$ are expressed as,

$$
\boldsymbol{c}^{s}=\left[\begin{array}{c}
c_{1}^{e 1, s} \\
c_{1}^{e 2, s} \\
c_{1}^{e 3, s} \\
\vdots \\
c_{1}^{e(N-1), s} \\
c_{1}^{e N, s} \\
c_{2}^{e N, s}
\end{array}\right]=\left[\begin{array}{c}
c_{1}^{s} \\
c_{2}^{s} \\
c_{3}^{s} \\
\vdots \\
c_{N-1}^{s} \\
c_{N}^{s} \\
c_{N+1}^{s}
\end{array}\right]
$$

$$
\dot{\boldsymbol{c}}^{s}=\left[\begin{array}{c}
\dot{c}_{1}^{e 1, s} \\
\dot{c}_{1}^{e 2, s} \\
\dot{c}_{1}^{e 3, s} \\
\vdots \\
\dot{c}_{1}^{e(N-1), s} \\
\dot{c}_{1}^{e N, s} \\
\dot{c}_{2}^{e N, s}
\end{array}\right]=\left[\begin{array}{c}
\dot{c}_{1}^{s} \\
\dot{c}_{2}^{s} \\
\dot{c}_{3}^{s} \\
\vdots \\
\dot{c}_{N-1}^{s} \\
\dot{c}_{N}^{s} \\
\dot{c}_{N+1}^{s}
\end{array}\right]
$$

where $c_{n}^{s}=c_{1}^{e n, s}=c_{2}^{e(n-1), s}$ and $\dot{c}_{n}^{s}=\dot{c}_{1}^{e n, s}=\dot{c}_{2}^{e(n-1), s}$. 
$\boldsymbol{Q}^{s}=\left[\begin{array}{c}Q_{1}^{e 1} \\ Q_{2}^{e 1}+Q_{1}^{e 2} \\ Q_{2}^{e 2}+Q_{1}^{e 3} \\ \vdots \\ Q_{2}^{e(N-2)}+Q_{1}^{e(N-1)} \\ Q_{2}^{e(N-1)}+Q_{1}^{e N} \\ Q_{2}^{e N}\end{array}\right]=\left[\begin{array}{c}Q_{1} \\ 0 \\ 0 \\ \vdots \\ 0 \\ 0 \\ Q_{N+1}\end{array}\right]$

where $Q_{2}^{e n, s}+Q_{1}^{e(n+1), s}=0$ as "mass-in=mass-out" at the boundaries of elements.

As a result, Equation [S.5] for 1-element system is modified for $N$-element system as,

$$
\left(\boldsymbol{L}^{s}+\boldsymbol{M}^{s}\right) \boldsymbol{c}^{s}+\boldsymbol{E} \dot{\boldsymbol{c}}^{s}=\boldsymbol{Q}^{s}
$$

For the time marching scheme for a 1D transient equation, Reddy ${ }^{37}$ implements $\alpha$-family of approximation.

$c_{n}^{s+1}=c_{n}^{s}+\Delta t\left[(1-\alpha) \dot{c}_{n}^{s}+\alpha \dot{c}_{n}^{s+1}\right]$

where $\alpha=2 / 3$ for Galerkin method. To rearrange Equation [S.15] yields, ${ }^{37}$

$$
c_{n}^{s+1}=c_{n}^{s}+a_{1} \dot{c}_{n}^{s+1}+a_{2} \dot{c}_{n}^{s}
$$

where $a_{1}=\alpha \Delta t$ and $a_{2}=(1-\alpha) \Delta t$.

At $t=t_{s+1}$, Equation [S.14] can be written as,

$$
\left(\boldsymbol{L}^{s+1}+\boldsymbol{M}^{s+1}\right) \boldsymbol{c}^{s+1}+\boldsymbol{E} \dot{\boldsymbol{c}}^{s+1}=\boldsymbol{Q}^{s+1}
$$

To implement the technique used by Reddy $^{37}$ to yield an equation to relate Equations [S.14] and [S.17], Equations [S.14] and [S.17] are rewritten as, 


$$
\begin{aligned}
& \boldsymbol{E} \dot{\boldsymbol{c}}^{s}=\boldsymbol{Q}^{s}-\left(\boldsymbol{L}^{s}+\boldsymbol{M}^{s}\right) \boldsymbol{c}^{s} \\
& \boldsymbol{E} \dot{\boldsymbol{c}}^{s+1}=\boldsymbol{Q}^{s+1}-\left(\boldsymbol{L}^{s+1}+\boldsymbol{M}^{s+1}\right) \boldsymbol{c}^{s+1}
\end{aligned}
$$

To multiply matrix $\boldsymbol{E}$ to each side of Equation [S.16] yields,

$$
\boldsymbol{E} \boldsymbol{c}^{s+1}=\boldsymbol{E} \boldsymbol{c}^{s}+a_{1} \boldsymbol{E} \dot{\boldsymbol{c}}^{s+1}+a_{2} \boldsymbol{E} \dot{\boldsymbol{c}}^{s}
$$

Substituting Equations [S.18] and [S.19] into Equation [S.20], and then rearranging it gives Equation [S.21].

$$
\left[\boldsymbol{E}+a_{1}\left(\boldsymbol{L}^{s+1}+\boldsymbol{M}^{s+1}\right)\right] \boldsymbol{c}^{s+1}=\left[\boldsymbol{E}-a_{2}\left(\boldsymbol{L}^{s}+\boldsymbol{M}^{s}\right)\right] \boldsymbol{c}^{s}+\left(a_{1} \boldsymbol{Q}^{s+1}+a_{2} \boldsymbol{Q}^{s}\right)
$$

In Equation [S.21], the time-independent matrices $\boldsymbol{L}$ and $\boldsymbol{M}$ in Reddy's ${ }^{37}$ original equation are modified to time-dependent matrices $\boldsymbol{L}^{\boldsymbol{s}}$ and $\boldsymbol{M}^{\boldsymbol{s}}$, respectively.

The technique that Reddy ${ }^{36,37}$ used to eliminate the first rows of matrices and vectors, and the first columns of matrices for a time-independent steady state system can also be applied to this 1D transient system. As the concentration at gas-heavy oil interface, $c_{1}^{s}$, i.e., $c_{\text {sat }}$ in Equation [2c] for DVA test or $c_{s a t}[p(t)]$ in Equation [2d] for pressure decay test, is able to be calculated from the flash calculation and volume-translated PR-EOS for every temporal step, $t_{s}$, the first rows in all matrices and vectors in Equations [S.14] and [S.17], and thus in Equation [S.21] can be eliminated. Also, the first columns in matrices, $\boldsymbol{E}, \boldsymbol{L}$, and $\boldsymbol{M}$ are able to be deleted. Furthermore, vector $\boldsymbol{Q}$ shall be replaced by vector $\boldsymbol{F}$ to combine the terms emerged at the right-hand side of Equation [S.21] due to such eliminations of the rows and columns. The reduction of matrices and vectors alters Equation [S.21] to become Equation [25]. 


\section{Pressure-time Relation of Pressure Decay Test from Li and Yang (2016)}

The pressure-time relation for $\mathrm{CO}_{2}$ diffusion test under $3741-3371 \mathrm{kPa}$ for 168 hours of test time is expressed by Equation [S.22]. ${ }^{11}$

$p(t)=81.7014 e^{-t / 19558.7545}+367.8442 e^{-t / 455580.8656}+3276.3647$

where $p(t)$ is decaying pressure in $\mathrm{kPa}$ at time $t$ in s. Equation [S.22] can be used to compute the equilibrium pressure as $3276.4 \mathrm{kPa}$. 